\title{
BMJ Open Integration of oral health into primary care: a scoping review protocol
}

To cite: Emami E, Harnagea $\mathrm{H}$, Girard F, et al. Integration of oral health into primary care: a scoping review protocol. BMJ Open 2016;6:e013807.

doi:10.1136/bmjopen-2016013807

- Prepublication history for this paper is available online. To view these files please visit the journal online (http://dx.doi.org/10.1136/ bmjopen-2016-013807).

Received 12 August 2016 Revised 22 September 2016 Accepted 23 September 2016

CrossMark

For numbered affiliations see end of article.

Correspondence to Dr Elham Emami; elham.emami@umontreal.ca

\section{ABSTRACT}

Introduction: Integrated care has been introduced as a means of improving health outcomes and access to care, and reducing the cost of healthcare. Despite its importance, the integration of oral health into primary care is still an emerging healthcare pathway. This scoping review protocol has been developed and funded by the Canadian Institutes of Health Research to provide an evidence-based synthesis on a primary oral healthcare approach and its effectiveness in improving oral health outcomes.

Methods and analysis: The 6-stage framework developed by Levac et al underpins this scoping review. We will identify relevant existing theories, programmes and original research through a comprehensive and systematic search of electronic databases such as OVID (MEDLINE, EMBASE, Cochrane databases), NCBI (PubMed), EBSCOhost (CINAHL), ProQuest, Databases in Public Health, Databases of the National Institutes of Health (health management and health technology) and relevant organisational websites and other sources of grey literature. All types of studies from 1978 to May 2016 in the French and English languages will be included. Using the Rainbow conceptual model of integrative primary care, a qualitative descriptive approach and thematic analysis will be used to synthesise the literature. Implementing novel healthcare models necessitates identifying barriers, sharing knowledge and delivering information. The integration of oral healthcare into primary care is an approach that promotes breaking the boundaries separating oral healthcare professionals and primary care. It creates opportunities for the dental workforce to become more involved in community-based practice and to assume shared responsibility with healthcare professionals to address the unmet oral health needs of those experiencing vulnerability and marginalisation.

Ethics and dissemination: The scoping study has received approval from the Université de Montréal's Institutional Review Board (\#14-097-CERES-D). The findings will be disseminated through publications and presentations in provincial, national and international research symposiums and professional meetings.

\section{INTRODUCTION}

Canada, similar to other parts of the developed world, has gone through major

\section{Strengths and limitations of this study}

- This scoping review will benefit various stakeholders by expanding the practical and theoretical knowledge on the integration of oral health into primary care.

- The review will identify knowledge gaps and the need for further research.

- The scoping review has consolidated methodology by using a theoretical framework of integrated care combined with an inductive thematic analysis.

- The engagement of key stakeholders at all stages will help to address any potential gap in conducting this review.

- The review will not include any quality assessment and grading of evidence since this is not part of the methodology used.

healthcare reforms with the intent to significantly improve the service organisation. ${ }^{1-4}$ This evolution stemmed from an 'epidemiological transition', as the challenges faced by the healthcare system were redefined, whereby economic compression, cultural diversity of communities, increasing burden of the ageing population and associated chronic conditions gradually gained more importance. ${ }^{5}$ This epidemiological revolution forces the merging of traditional healthcare approaches to a multidimensional approach, by means of innovative integration techniques. Integrated care models emphasise the importance of providing services that meet the needs of people with multiple health and social problems. ${ }^{6-9}$

Integrated care is defined as 'bringing together inputs, delivery, management and organisation of services related to diagnosis, treatment, care, rehabilitation and health promotion'. ${ }^{10}$ The integration of services fits into a public health perspective and follows the WHO adopted 'six building blocks' (leadership/governance, healthcare financing, health workforce, technologies, information and research, service delivery) for strengthening the health system. ${ }^{11}$ This 
perspective calls organisations to create new ways of disease prevention and health promotion by establishing a network of key health actors whose interventions will be more effective if they are integrated. ${ }^{12}$ In this system, all the health service providers intervene in a crucial network to increase their capacity to have a positive effect on the determinants of health. ${ }^{13}$ The integrated primary care concept encourages bringing together health professionals from different disciplines and sectors to improve service efficiency and quality of health services and thus to reduce differences in access and usage of services between geographical and socioeconomic groups. ${ }^{14}$ It puts an accent on access, quality and efficiency of care, and serves as a well-coordinated and proactive health and social services system, which is sensitive to the needs, experiences and culture of healthcare consumers. ${ }^{15} 16$ Integrated systems of care have been demonstrated to be beneficial from a clinical, policy and economic perspective. ${ }^{17}$

The integration of oral health into primary care or the primary oral healthcare (POHC) approach has been recognised as a promising solution for the challenges of dental service provision, especially for disadvantaged populations. ${ }^{18-20}$ Rooted in the Alma-Ata Declaration (1978), primary healthcare has been introduced to increase the effectiveness of healthcare systems. ${ }^{20}$ In fact, for many members of disadvantaged communities, primary healthcare and public health services are the first contact with the healthcare system. ${ }^{21-25}$ Although the integration of oral health into primary care has been realised in a few countries such as Brazil and the $\mathrm{UK}$, in several developing and industrial countries the dental workforces are still not fully present in the community primary healthcare systems. ${ }^{26}$ As a matter of fact, historically in countries such as Canada and the USA, the private sector has been responsible for providing oral healthcare services. ${ }^{27} 28$ This mode of service does not ensure equity in oral healthcare and services, mainly because of two factors: the high costs of dental care and the shortage of dentists offering services to vulnerable and disadvantaged communities such as rural, remote and aboriginal populations. ${ }^{29} 30$ In fact, a myriad of problems can exist when looking at access to necessary dental care. These include a lack of public dental care, a lack of appropriate facilities for dental care of patients with medical and social needs, and a lack of screening for and monitoring of oral chronic diseases. ${ }^{31-33}$ During the past decade, a number of oral health integrated primary healthcare models have emerged worldwide to address these challenges and the WHO policies give priority to this approach. ${ }^{34-37}$

However, the concept of integrating oral health with primary care is still unclear and hampered by a lack of systematic understanding. Therefore, the objective of this paper is to present a protocol for a scoping study which aims to map out the literature on the POHC approach and to produce an evidence-based synthesis of the theoretical and applied concepts of oral health integrated primary care and its effectiveness. Furthermore, the scoping review will serve to guide the future research on this topic. This evidence will be transferred to appropriate knowledge users to enhance policy decisions and practice change through encouraging collaboration. This will definitively lead to patients' empowerment.

\section{METHODS AND ANALYSIS}

The study will be conducted using Levac et $a l^{38}$ sixstage methodological framework: (1) identifying the research question, (2) searching for relevant studies, (3) selecting studies, (4) charting the data and collating, (5) summarising and reporting the results and (6) stakeholders' consultations to inform the review.

\section{Identifying the research question}

On the basis of the Canadian Institute of Health Research integrated knowledge translation model, ${ }^{39}$ we have engaged expert researchers, policymakers and knowledge users in the entire process of this project to foster mutual understanding across the key stakeholders. At this stage, a set of questions were formed to be answered by the scoping study:

1. What do we already know about the POHC model: common and specific characteristics worldwide?

2. Are there any public health policies in regard to the integration of oral healthcare into primary care and worldwide?

3. To what extent can the POHC models improve the oral health and the health of the vulnerable population?

4. What are the barriers and the facilitators of an integrated oral health primary care in different settings?

5. What types of research have been conducted on the subject and what were their goals?

6. What are the gaps in knowledge?

\section{Search strategy}

The detailed search strategy will be designed with the help of an expert librarian at Université de Montreal, using specific MeSH terms and keywords to assure the accuracy and sensitivity of the search to capture the relevant literature. The search will be conducted using various electronic platforms such as: OVID (MEDLINE, EMBASE, Cochrane databases), NCBI (PubMed), EBSCOhost (CINAHL), ProQuest, Databases in Public Health, Databases of the National Institutes of Health (health management and health technology), Health Services and Sciences Research Resources, Health Services Research \& Health Care Technology, Health Services Research Information Central, Health Services Research Information Portal, Health Services Technology Assessment Texts, and Healthy People 2020 Structured Evidence Queries. Data will also be extracted from official reports and websites of various relevant organisations including professional associations, different 
Canadian institutions involved in primary healthcare research, as well as organisations such as the WHO and the International Dental Federation. A manual search of the bibliography of selected articles will be used to complement research and to identify grey literature. The search strategy will be revised and adapted for each of the electronic databases.

\section{Study selection and data extraction}

Publications in English or French from 1978 (Alma-Ata Declaration) to April 2016 will be reviewed. We will include all research studies irrespective of study design in which the integration of oral health into primary care is the primary focus of the publication. We will exclude publications including commentaries, editorials and individual points of view. Two researchers (EE, HH) will independently screen the titles and abstracts of each citation and will identify eligible articles for full review. Disagreement between reviewers will be discussed and resolved by consensus. All potential relevant studies will be retained for full-text assessment. Data extraction will be conducted independently by the same reviewers using a data extraction form. The data extraction form will be designed according to the study conceptual framework.

\section{CONCEPTUAL FRAMEWORK}

The rainbow model has been introduced in 2013 by Valentijn $e t a t^{40}$ to provide guidance to explain the mechanisms of the integration and to facilitate the measurement of integration outcomes. This conceptual framework is based on the integrative functions of primary care and includes three level-specific dimensions: clinical integration (microlevel), organisational integration (mesolevel) and system integration (macrolevel). Furthermore, in this multilevel model, functional and normative integration assures a link between the other three dimensions.

According to the American Medical Association, clinical integration is 'the means to facilitate the coordination of patient care across conditions, providers, settings and time in order to achieve care that is safe, timely, effective, efficient, equitable and patientfocused'. ${ }^{41}$ This dimension of integrated care ensures individualised and coordinated care and requires a multidisciplinary team governing structure. The organisational integration represents the interorganisational relationships across the entire care continuum (horizontal and vertical integration) and assures the populationbased care. ${ }^{40}$ At the macrolevel, system integration combines structures, processes and techniques to respond to the needs of individuals and populations. ${ }^{40}$

\section{Data charting and collating}

Charting tables using Excel tools will be used to extract the data. To assure the consistency of the data extraction and coding, this stage will be conducted by the two reviewers.

\section{Summarising and reporting the results}

A qualitative descriptive approach will be used to synthesise the literature. A thematic analysis will be conducted and linked to the five dimensions of the integrated care included in the study conceptual framework. ${ }^{42}$ All emerging themes, which could not be classified according to the framework dimensions, will be analysed to expand the broad and the breath of the analysis.

\section{Stakeholder consultations}

This last stage will allow the engagement of the stakeholders in this scoping study. It will provide constructive feedback on the scoping study research questions, process and results. Furthermore, it represents an ideal strategy to ensure knowledge translation. The stakeholders include representatives of academic healthcare organisations (AC, RV), policy decision-makers (MC) and healthcare professionals $(\mathrm{JW})$.

\section{ETHICS AND DISSEMINATION}

The scoping study has received approval from the Université de Montréal's Institutional Review Board (\#14-097-CERES-D).

In this paper, we present a protocol for a scoping review designed systematically to identify the breadth of literature in the area of POHC. Integrated knowledge translation research bridges the worlds of research, policy and practice, strengthens the links between key stakeholders and knowledge users and thereby leads to tangible actions. $^{39}$

Recently, the American Academy of Family Physicians has supported the integration of oral health into primary care to reduce the burden of oral health diseases and to meet the needs of people with multiple health and social problems. ${ }^{43}$ This consensus was supported by an interdisciplinary team of health and oral healthcare providers, the representatives of professional associations and public health advocates as well as policymakers and care consumers. ${ }^{43}$ However, the implementation of this approach may encounter various challenges. ${ }^{44}{ }^{45}$ Furthermore, its concepts, the related models and policies need to be explored comprehensively.

Scoping reviews are used to understand complex phenomena of interest. Generally, a scoping review aims to: (1) map the literature in an area of interest, (2) determine the usefulness of conducting a systematic review, (3) summarise and disseminate research findings and (4) capitalise future research capacity by identification of research gaps. ${ }^{38} 46$ The findings will be disseminated through publications and presentations in provincial, national and international research symposiums and professional meetings.

Providing evidence on the effectiveness of POHC models, and identification of its challenges, facilitators and barriers will enable the contextualisation of the concept. Furthermore, the provided recommendations 
will raise awareness of policymakers and healthcare professionals from other disciplines in regard to the importance of the integration of oral health into primary care. Finally, this knowledge transfer will sensitise the oral healthcare professionals and dental workforce to become more involved in community-based practice and to assume their shared responsibility with healthcare professionals to address the unmet oral health needs of marginalised populations. ${ }^{47} 48$

\section{CONCLUSION}

We expect that the knowledge generated from this research study will help to promote better access to oral healthcare and improvement of oral health for disadvantaged populations.

\section{Author affiliations}

${ }^{1}$ Faculty of Dentistry, School of Public Health, Université de Montréal, McGill University, Montreal, Quebec, Canada

2École de santé publique, Université de Montréal, Montréal, Québec, Canada ${ }^{3}$ Faculté de médecine dentaire, Université de Montréal, Montréal, Québec, Canada

${ }^{4}$ Faculty of Dentistry, McGill University, McGill College, Montreal, Quebec, Canada

${ }^{5}$ Public Health Agency of Canada, Ottawa, Ontario, Canada

${ }^{6}$ Department of Family Medicine, Gatineau Integrated Center of Health and Social Services, Shawville, Québec, Canada

${ }^{7}$ École de travail social, Université de Sherbrooke, Sherbrooke, Québec, Canada

Acknowledgements The authors would like to gratefully acknowledge the help of Dupont Patrice (librarian, Université de Montréal) for the design of the search strategy.

Contributors All authors have made significant intellectual contributions to this scoping review protocol. As a principal investigator, EE contributed largely to the conception of the review project and the development of the protocol. She provided guidance to the research team members and secured funds for the study. She will coordinate all aspects of the study and play an essential role in the analysis and interpretation of the results. HH conducted the preliminary literature review and collaborated in the protocol development and writing of the protocol. She designed the search strategy of the scoping review with an expert librarian at Université de Montréal. She will extract the data with $\mathrm{EE}$ and will contribute to the study analysis and interpretation with the collaboration of other team members. FG, YC, CPB and AC were involved in the protocol development and will contribute to data analysis and data interpretation. RV, MC and JW are the main stakeholders and will provide the needed feedback at various stages of the study. All authors revised the manuscript and approved the final version.

Funding This study is funded by a Knowledge Synthesis Grant from the Canadian Institutes for Health Research (grant number: KRS-138220).

Competing interests None declared

Provenance and peer review Not commissioned; externally peer reviewed.

Open Access This is an Open Access article distributed in accordance with the Creative Commons Attribution Non Commercial (CC BY-NC 4.0) license, which permits others to distribute, remix, adapt, build upon this work noncommercially, and license their derivative works on different terms, provided the original work is properly cited and the use is non-commercial. See: http:// creativecommons.org/licenses/by-nc/4.0/

\section{REFERENCES}

1. Contandriopoulos D, Brousselle A, Breton M, et al. Nurse practitioners, canaries in the mine of primary care reform. Health Policy 2016;120:682-9.
2. Deber R. Health care reform: lessons from Canada. Am J Public Health 2003;93:20-4.

3. Tuohy C. Accidental logics: the dynamics of change in the health care arena in the United States, Britain, and Canada. New York: Oxford University Press, 1999.

4. Hutchison B, Abelson J, Lavis J. Primary care in Canada: so much innovation, so little change. Health Aff (Millwood) 2001;20:116-31.

5. Omran A. The epidemiological transition: a theory of the epidemiology of population change. Milbank Mem Fund $Q$ 1971;83:731-57.

6. King M, Smith A, Gracey M. Indigenous health part 2: the underlying causes of the health gap. Lancet 2009;374:76-85.

7. Leatt $P$, Pink GH, Guerriere M. Towards a Canadian model of integrated healthcare. Healthc Pap 2000;1:13-36.

8. Bedos C, Loignon C, Brodeur J, et al. Pauvreté et accès aux services buccodentaires: entre droit et éthique. Toulouse, France: Congrès mondial de droit médical, 2006.

9. Bedos C, Loignon C, Landry A, et al. Providing care to people on social assistance: how dentists in Montreal, Canada, respond to organisational, biomedical, and financial challenges? A qualitative study in Montreal, Canada. BMC Health Serv Res 2014;14:472.

10. World Health Organisation. Integrated health services-what and why. Making health systems work. World Health Organisation, 2008.

11. World Health Organization. Everybody business: strengthening health systems to improve health outcomes: WHO's framework for action. Geneva, Switzerland: World Health Organization, 2007.

12. Kodner DL, Spreeuwenberg C. Integrated care: meaning, logic, applications and implications-a discussion paper. Int $J$ Integr Care 2002;2:e12.

13. Couturier $Y$, Gagnon D, Belzile L, et al. La coordination en gérontologie. Montréal, Québec, Canada: Les Presses de l'Université de Montréal, 2013.

14. World Health Organization European Office for Integrated Health Care Services. Integrated care. working definition. World health organization, 2001.

15. Kodner D, Kyriacou C. Fully integrated care for frail elderly: two American models. Int J Integr Care 2000;1:1e08.

16. Contandriopuolos A, Denis J, Touati N, et al. Intégration de soins: dimensions et mise en oeuvre. Ruptures Revue Transdisciplinaire Sante 2001:8:38-52.

17. Hollander $\mathrm{MJ}$, Kadlec $\mathrm{H}$, Hamdi R, et al. Increasing value for money in the Canadian healthcare system: new findings on the contribution of primary care services. Healthc Q 2009:12:32-44.

18. Patel J, Hearn L, Gibson B, et al. International approaches to Indigenous dental care: what can we learn? Aust Dent $J$ 2014:59:439-45.

19. World Health Organization. The World Health report 2008: primary health care now more than ever. Geneva: World Health Organization, 2008

20. World Health Organization UNICEF. Report of the conference on Primary Health Care. Alma-Ata, USSR: World Health Organization, 1978.

21. Aday L. At risk in America: the health and health care needs of vulnerable populations in the United States. 2nd edn. San Francisco. 2001.

22. Beland F, Hollander MJ. Integrated models of care delivery for the frail elderly: international perspectives. Gac Sanit 2011;25(Suppl 2):138-46.

23. Brennan DS, Spencer AJ, Slade GD. Provision of public dental services in urban, rural and remote locations. Community Dent Health 1996;13:157-62.

24. Butler GC. Delivery of health care in Northern Canada. Can J Ophthalmol 1973;8:188-95.

25. Fellona M, DeVore LR. Oral health services in primary care nursing centers: opportunities for dental hygiene and nursing collaboration. J Dent Hyg 1999;73:69-77.

26. Ministry of Health. Environmental scan: oral health services in British Columbia for First Nations and Aboriginal children aged 0-7 years. Victoria, BC: Ministry of Health, 2012.

27. American Dental Association. Breaking down barriers to oral health for all Americans: the role of workforce. J Calif Dent Assoc 2011;39:491-502.

28. Allison $\mathrm{P}$, Allington $\mathrm{C}$, Stern J. L'accès aux soins dentaires des personnes défavorisées au Québec - problématique et pistes de solutions: Faculté de médecine dentaire, McGill University. 2004.

29. Lawrence HP, Binguis D, Douglas J, et al. Oral health inequalities between young Aboriginal and non-Aboriginal children living in Ontario, Canada. Community Dent Oral Epidemiol 2009;37:495-508. 
30. Lawrence $\mathrm{H}$, Romanetz M, Rutherford L, et al. Oral health of aboriginal preschool children in Northern Ontario. Probe 2004;38:172-90.

31. Health Canada. Inuit Oral Health Survey Report: 2008-2009. Ottawa, Ontario, 2011.

32. Emami E, Khiyani M, Habra C, et al. Mapping Quebec dental workforce: ranking rural oral health disparities. Rural Remote Health 2016;16:3630.

33. Emami E, Wootton J, Galarneau C, et al. Oral health and access to dental care: a qualitative exploration in rural Quebec. Can J Rural Med 2014;19:63-70.

34. Cohen LA. Expanding the physician's role in addressing the oral health of adults. Am J Public Health 2013;103:408-12.

35. Cane R, Walker J. Rural public dental practice in Australia: perspectives of Tasmanian government-employed dentists. Aust $J$ Rural Health 2007;15:257-63.

36. Petersen $P$. Challenges to improvement of oral health in the 21st century - the approach of the WHO Global Oral Health Programme. Int Dent J 2004;56:329-43.

37. Petersen $\mathrm{PE}$, Bourgeois $\mathrm{D}$, Ogawa $\mathrm{H}$, et al. The global burden of oral diseases and risks to oral health. Bull World Health Organ 2005;83:661-9.

38. Levac D, Colquhoun H, O'Brien KK. Scoping studies: advancing the methodology. Implement Sci 2010;5:1-9.

39. Canadian Institutes of Health Research. Guide to knowledge translation planning at ClHR. Governement of Canada, 2015.
40. Valentijn PP, Schepman SM, Opheij W, et al. Understanding integrated care: a comprehensive conceptual framework based on the integrative functions of primary care. Int J Integr Care 2013;13:e010.

41. American Hospital Association. Clinical Integration-The Key to Real Reform [database on the Internet] 2010. http://www.aha.org/ research/reports/tw/10feb-clinicinteg.pdf (accessed 10 Oct 2016).

42. Wodchis W, Williams A, Merry G. Integrating care for persons with chronic health and social needs. Creating strategic change in Canadian healthcare; Ontario, Canada. 2014.

43. Brownlee B. Oral health integration in the patient-centered medical home environment: case studies from community health centers. Qualis Health/DentaQuest Foundation, 2012.

44. Vandamme K, Opdebeeck H, Naert I. Pathways in multidisciplinary oral health care as a tool to improve clinical performance. Int J Prosthodontics 2006;19:227-35.

45. Badenbroek IF, Stol DM, Nielen MM, et al. Design of the INTEGRATE study: effectiveness and cost-effectiveness of a cardiometabolic risk assessment and treatment program integrated in primary care. BMC Fam Pract 2014;15:90

46. Arksey H, O'Malley L. Scoping studies: towards a methodological framework. Int J Soc Res Meth 2005:8:19-32.

47. Vanasse A, Courteau J, Cohen AA, et al. Rural-urban disparities in the management and health issues of chronic diseases in Quebec (Canada) in the early 2000s. Rural Remote Health 2010;10:1548-53.

48. Emami E, Feine JS. Focusing on oral health for the Canadian rural population. Can J Rural Med 2008;13:36-8. 